\title{
Radome Design for Hat-Fed Reflector Antenna
}

\author{
Erik Geterud \\ *, Jian Yang*, Tomas Ostling ${ }^{\dagger}$, \\ * Department of Signals and Systems, Chalmers University of Technology, Gothenburg, Sweden \\ Email: erik.geterud@chalmers.se; jian.yang@chalmers.se \\ $\dagger$ Arkivator AB, Gamlestadsvagen 303, SE-415 25 Gothenburg, Sweden \\ Email: tomas.ostling@arkivator.se
}

\begin{abstract}
We present the design of a low loss homogenous radome to house a hat-fed reflector antenna for the $\mathrm{Ku}$ satellite communication band. The radome is made of polycarbonate and can easily be vacuum formed in series. Simulated and measured data show that the radome has a low reflection coefficient and low ohmic loss, as well as very little effect on the antenna radiation patterns over the required frequency band.
\end{abstract}

\section{INTRODUCTION}

The waveguide self-supported hat feed for reflector antennas has many advantages: low cross polarization level due to the similar E- and H-plane radiation functions, low far-out sidelobes, low blockage as no struts are needed to support the feed, and low ohmic loss since the electronic devices (such as transceiver) can be placed directly behind the reflector [1][7]. A recent development is the use of the hat-fed reflector antenna for satellite communication [8]. This paper focuses on the radome design for a $53 \mathrm{~cm}$ diameter hat-fed reflector antenna in the $\mathrm{Ku}$ satellite band of $10.75-12.75 \mathrm{GHz}(\mathrm{Rx})$ and 13.75-14.5 GHz (Tx). As the antenna will be used outdoors, the radome shall protect from harsh environment including gale winds, heavy rain, hail, snow and UV radiation.

\section{RADOME DESIGN}

Radomes are generally constructed of either single layer plastics or multiple layer sandwich structures.

Sandwich radomes have the advantages of high strength, low weight and may have wideband performance. The drawback is that they are more complex and costly to manufacture than single layer radomes.

For this application, we have chosen a single layer radome as it is robust enough for moderate radome sizes, low loss over the required frequency band and easy to manufacture in series by vacuum forming. The diameter of the radome is $66,4 \mathrm{~cm}$ and the material is chosen as polycarbonate. The dielectric permittivity of the polycarbonate is $\epsilon_{r}=2.9$ with a loss tangent $\tan \delta=0.005$.

The modeling of the radome with the hat-fed reflector antenna was done by using the 2D FDTD simulation software QuickWave V2D, shown in Fig. 1. As the antenna is axially symmetric, a symmetry plane is used, which reduces the computation time by a factor of two. A hemispherical radome was placed in front of the hat-fed reflector antenna and the reflection coefficient was analyzed with various radome

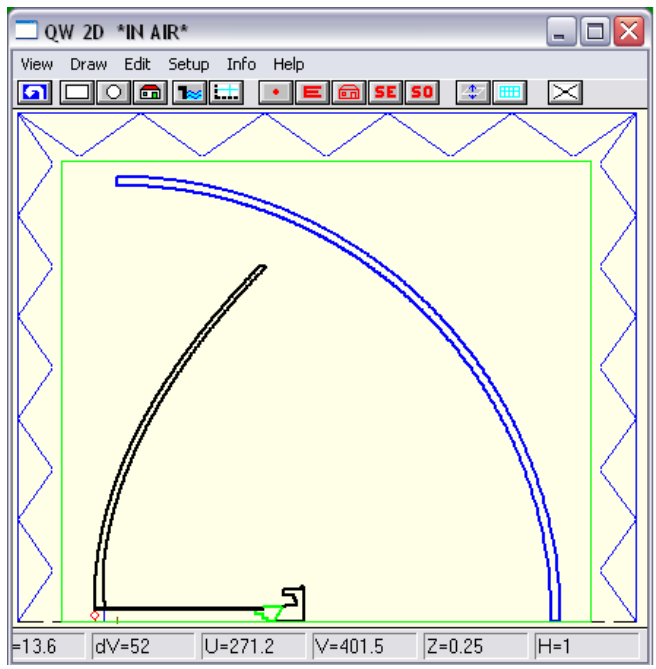

Fig. 1. Simulation model of radome enclosed hat-fed reflector antenna in QuickWave V2D.

thicknesses. The influence on reflection coefficient of the hatfed reflector antenna with varied radome thicknesses is shown in Fig. 2. After the analysis, the radome thickness in the axial direction was chosen to $7.1 \mathrm{~mm}$ corresponding to $\lambda / 2$ at the centre frequency $12.4 \mathrm{GHz}$. This configuration has the minimum effect on the reflection coefficient over the required frequency band.

A drawing of the full radome with the final dimensions are shown in Fig 3. This radome consists of three parts, a rigid bottom tray, a cylindrical mid section and a hemispherical top section. This shape makes it inherently strong and the volume is sufficient for the antenna to be able to freely move inside the radome and change pointing direction.

\section{MEASUREMENTS}

The measurements are made in the anechoic chamber at Chalmers University of Technology. The radome loss is measured by placing the radome between two corrugated feed horns and measuring $S_{21}$ with reference to air, shown in Fig. 4. We see that the loss (including the mismatch factor) is only $0.1 \mathrm{~dB}$ in the Tx band and less than $0.57 \mathrm{~dB}$ over the full band, shown in Fig. 5. The loss at the band edges is a consequence of using single layer technique over a wide band. The reflection 


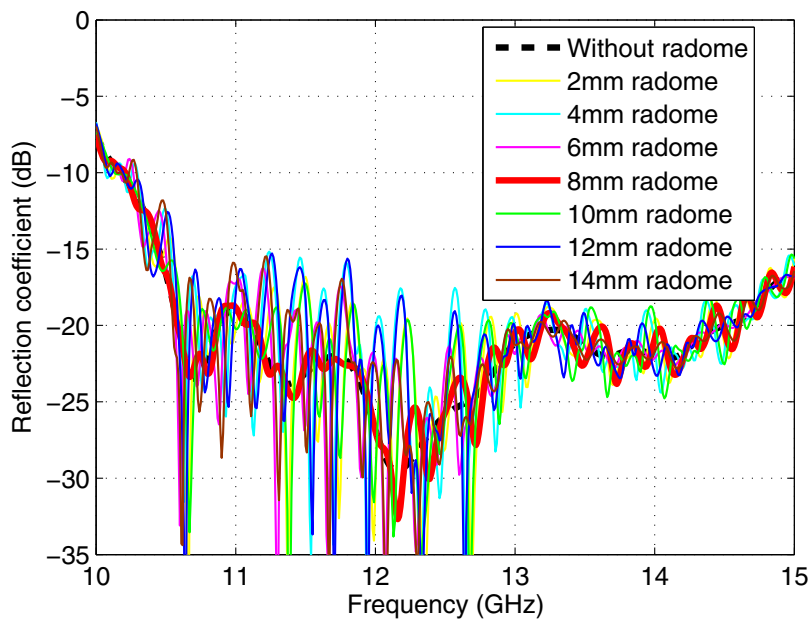

Fig. 2. Simulated reflection coefficient of hat-fed reflector antenna enclosed in radome of various thickness.

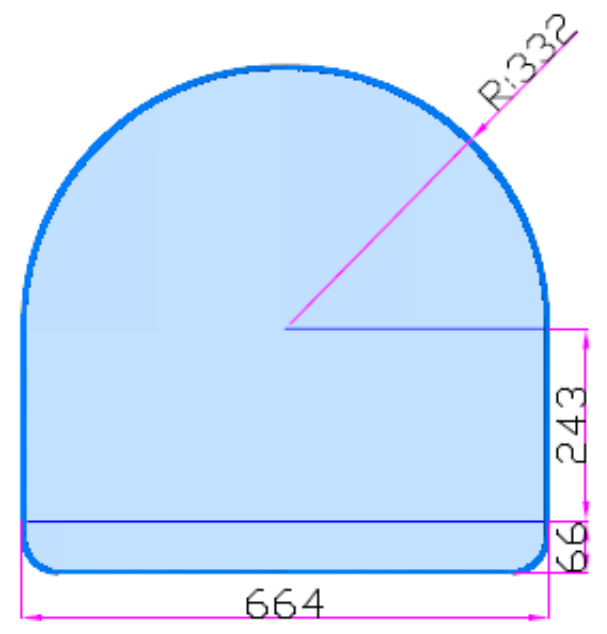

Fig. 3. Drawing of the full radome with dimensions in $\mathrm{mm}$.

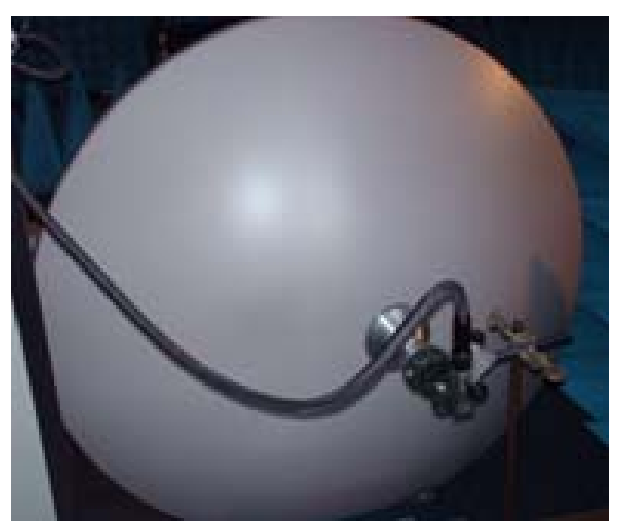

Fig. 4. Radome under test of reflection coefficient.

coefficient is measured and we see in Fig. 6 that there is a frequency shift in the response but the levels are similar,

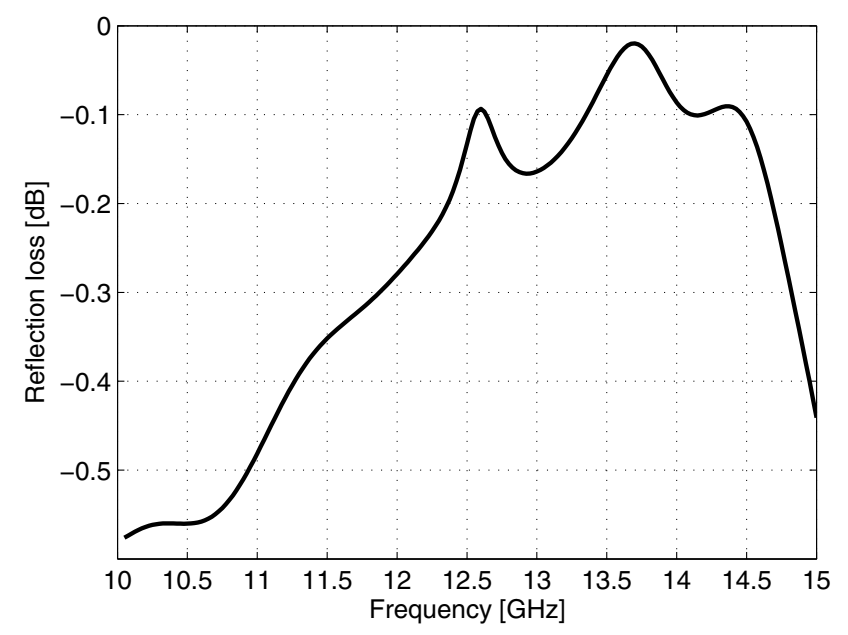

Fig. 5. Measured radome loss.

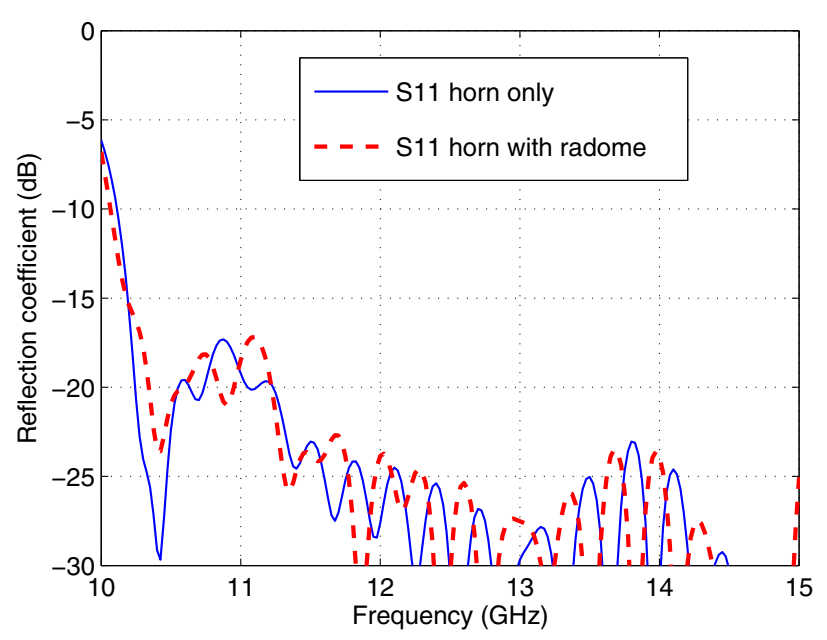

Fig. 6. Measured reflection coefficient of a corrugated horn without radome and when it touches directly on the radome.

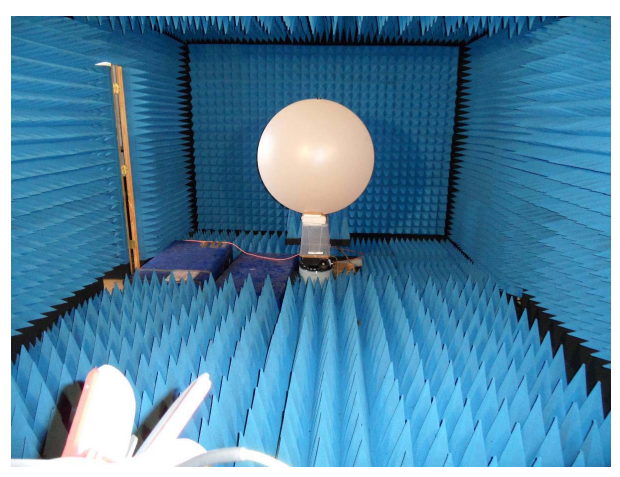

Fig. 7. Radome under test of reflection coefficient and radiation patterns.

showing that the radome has little effect on the performance.

The radome is thereafter mounted in front of the hat-fed 


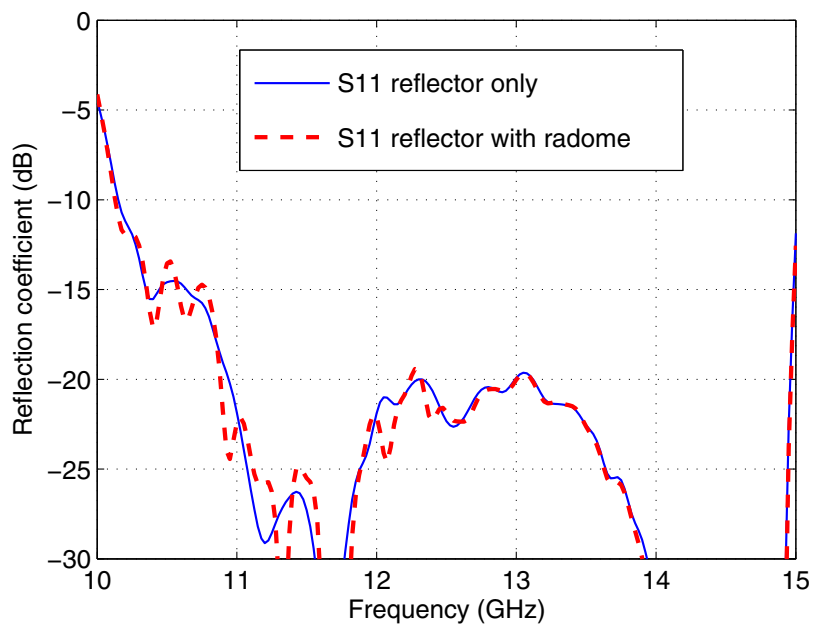

Fig. 8. Measured reflection coefficient of hat-fed reflector antenna with and without radome.

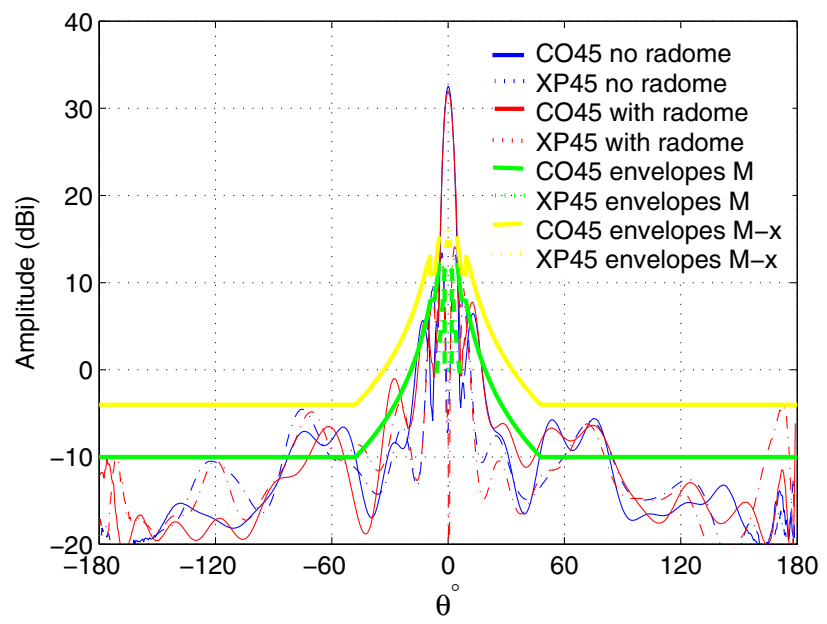

Fig. 9. Radiation patterns of hat fed reflector antenna with and without radome at $10.75 \mathrm{GHz}$.

reflector antenna and the reflection coefficient is measured; see Fig 7. Also in this setup we see in Fig. 8 that the effect of the radome on the reflection coefficient is very small.

The radiation patterns of the hat-fed reflector antenna with and without radome were also measured to see the influence on directivity, co- and cross polar sidelobe level. The radiation patterns were measured in the diagonal plane. In Figs. 911 , the sidelobe envelopes for satellite communication using the standards $M$ and $M-x$ have been included [9], [10]. At $10.75 \mathrm{GHz}$ we see that the sidelobe levels are slightly higher at +13 and -28 degrees but the levels are in general very little affected by the radome (Fig. 9). At $12.75 \mathrm{GHz}$, the patterns are still very similar. At \pm 30 degrees the sidelobes are slightly higher when the radome is included but at \pm 54 degrees the sidelobes are reduced (Fig. 10). Finally, at 14.50 $\mathrm{GHz}$ there is little difference between the level of the patterns

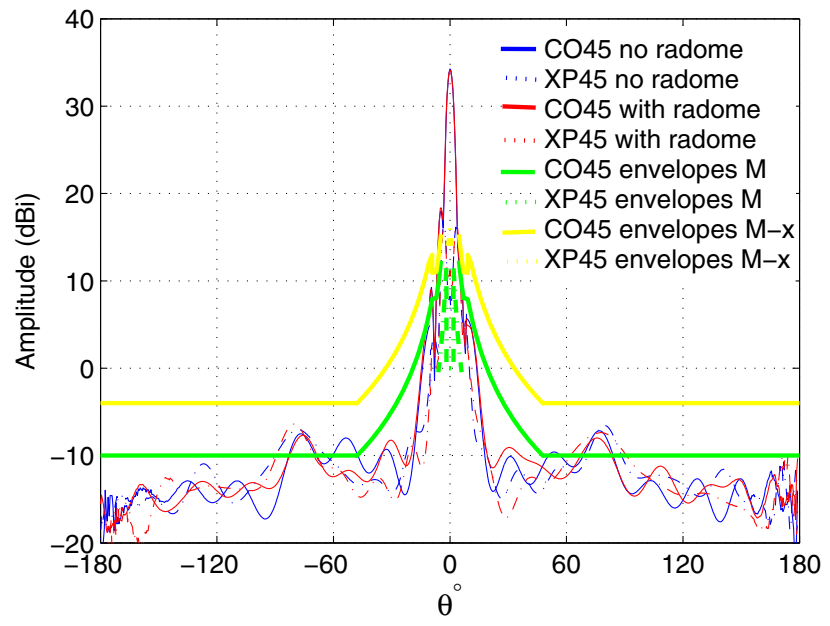

Fig. 10. Radiation patterns of hat fed reflector antenna with and without radome at $12.75 \mathrm{GHz}$.

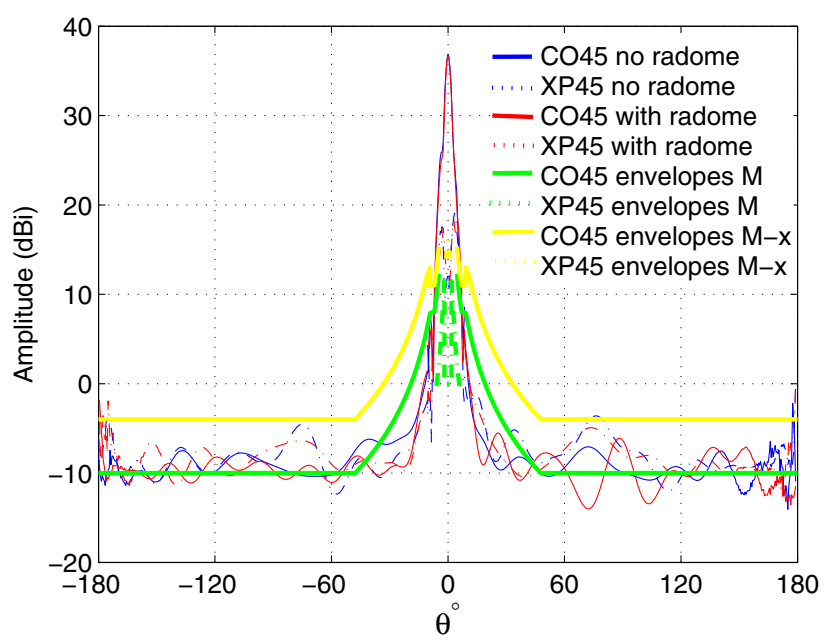

Fig. 11. Radiation patterns of hat fed reflector antenna with and without radome at $14.50 \mathrm{GHz}$.

but the sidelobe at \pm 75 degrees is sligthly higher when the antenna is not included (Fig. 11). There is only a slight reduction in directivity over the frequency band corresponding to the radome loss in Fig. 4. The radome has not had any significant effect on the cross-polar discrimination for any of the measured frequencies.

\section{CONCLUSION}

A single layer radome for the frequency band 10.75-14.50 $\mathrm{GHz}$ was simulated, manufactured and measures were done. The loss is low $(\leq 0.55 \mathrm{~dB} \mathrm{Rx}$ and $\leq 0.1 \mathrm{~dB} \mathrm{Tx})$ and the reflection coefficient is not significantly changed when the radome is placed in front on an antenna. Also, the radome has no significant effect on co- and cross-polar radiation patterns. As the radome is relatively small it will be rigid enough using a single layer technique and it can easily be manufactured in 
series using vacuum forming. For larger size radomes or if a wider frequency band of operation is required one can consider using sandwich radomes. However for this application the simplicity of a single layer radome made it favorable.

\section{ACKNOWLEDGMENT}

This work has been supported in part by The Swedish Foundation for Strategic Research (SSF) within the Strategic Research Center Charmant.

\section{REFERENCES}

[1] P.-S. Kildal, "The hat feed: a dual-mode rear-radiating waveguide antenna having low cross polarization," IEEE Trans. Antennas Propagat., vol. 35, no. 9, pp. 1010-1016, Sept. 1987.

[2] A. Moldsvor, M. Raberger, and P.-S. Kildal, "An efficient rectangular hat feed for linear polarization and low sidelobes," vol. 1. Digest of 1993 IEEE AP-S International Symposium, 28 June - 2 July 1993, pp. 270-273.

[3] J. Yang and P.-S. Kildal, "FDTD design of a Chinese hat feed for shallow mm-wave reflector antennas." Atlanta, Georgia: Proc. 1998 IEEE AP-S International Symposium, 21-26 June 1998, pp. 2046-2049.

[4] M. Yousefnia, A. Pirhadi, and M. Hakkak, "Analysis and design of parabolic hat feed antenna." Proc. 2005 IEEE AP-S International Symposium, 3-8 July 2005, pp. 650-653.

[5] M. Denstedt, T. Ostling, J. Yang, and P.-S. Kildal, "Tripling bandwidth of hat feed by genetic algorithm optimization." Hawai: 2007 IEEE AP-S International Symposium, 10-15 June 2007.

[6] W. Wei, J. Yang, T. Ostling, and T. Schafer, "A new hat feed for reflector antennas realized without dielectrics for reducing manufacturing cost and improving reflection coefficient," IET Microwaves Antennas Propagat., 2011.

[7] A. Yasin, J. Yang, and T. Ostling, "A compact dual-band feed for reflector antennas based on choke horn and circular eleven antenna," Antennas and Propagation, IEEE Transactions on, vol. 57, no. 10, pp. 3300-3302, 2009.

[8] E. Geterud, J. Yang, and T. Ostling, "Wide band hat-fed reflector antenna for satellite communications." Rome: 5th Eur. Conference on Antennas Propagat. (EuCAP2011), 11-15 April 2011.

[9] Eutelsat-M, "Earth station minimum technical and operational requirements. standard m, eess 502, issue 11-rev.1. oct. 2008."

[10] Eutelsat-M-x, "Conditions of operations of earth stations not fully complying with standard $\mathrm{m}$. nomenclature of standard $\mathrm{m}$ - $\mathrm{x}$. addendum to eess 502 . issue 1 , rev.1, oct. 2008." 